\title{
QCD FACTORIZATION FOR THE PION DIFFRACTIVE DISSOCIATION INTO TWO JETS
}

\author{
D. YU. IVANOV ${ }^{1,2}$ \\ ${ }^{1}$ Institut für Theoretische Physik, Universität Regensburg, \\ D-93040 Regensburg, Germany, \\ ${ }^{2}$ Institute of Mathematics, 630090 Novosibirsk, Russia, \\ E-mail: Dmitri.Ivanov@physik.uni-regensburg.de
}

\begin{abstract}
We report a detailed study of the process of pion diffraction dissociation into two jets with large transverse momenta. We find that the standard collinear factorization does not hold in this reaction. The structure of non-factorizable contributions is discussed and the results are compared with the experimental data. Our conclusion is that the existing theoretical uncertainties do not allow, for the time being, for a quantitative extraction of the pion distribution amplitude. (Talk presented at the Workshop on Exclusive Processes at High Momentum Transfer, Jefferson News, VA, May 15-18, 2002)
\end{abstract}

1. To our knowledge, the pion (and photon) diffraction dissociation into a pair of jets with large transverse momentum on a nucleon target was first discussed in ${ }^{1}$. Then the possibility to use this process to probe the nuclear filtering of pion components with a small transverse size was suggested in ${ }^{2}$. The A-dependence and the $q_{\perp}^{2}$-dependence of the coherent dijet cross section was first calculated in ${ }^{3}$. In the same work it was argued that the jet distribution with respect to the longitudinal momentum fraction has to follow the quark momentum distribution in the pion and hence provides a direct measurement of the pion distribution amplitude. Recent experimental data by the E791 collaboration ${ }^{4,5}$ indeed confirm the strong A-dependence which is a signature for color transparency, and are consistent with the predicted $\sim 1 / q_{\perp}^{8}$ dependence on the jet transverse momentum. Moreover, the jet longitudinal momentum fraction distribution turns out to be consistent with the $\sim z^{2}(1-z)^{2}$ shape corresponding to the asymptotic pion distribution amplitude which is also supported by an independent measurement of the pion transition form factor $\pi \gamma \gamma^{* 6}$.

After these first successes, one naturally asks whether the QCD description of coherent dijet production can be made fully quantitative. Two recent studies ${ }^{7}$ and ${ }^{8}$ address this question, with contradictory conclusions. 
Therefore we attempt to clarify the situation and develop a perturbative QCD framework for the description of coherent dijet production that would be in line with other known applications of the QCD factorization techniques. The results reported here have been obtained in collaboration with V.M. Braun, A. Schäfer and L. Szymanowski ${ }^{9,10}$.

2. The kinematics of the process is shown in Fig. 1. The momenta of

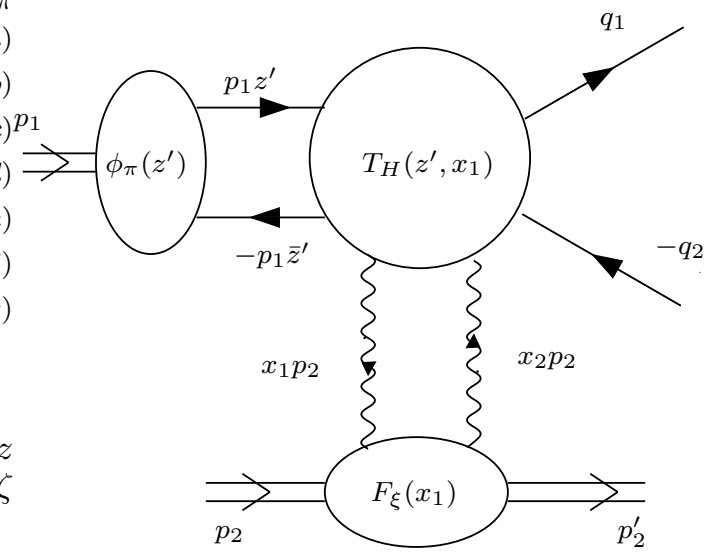

Figure 1. Kinematics of the coherent hard dijet production $\pi \rightarrow 2$ jets. The hard scattering amplitude $T_{H}$ contains at least one hard gluon exchange.

the incoming pion, incoming nucleon and the outgoing nucleon are $p_{1}, p_{2}$ and $p_{2}^{\prime}$, respectively. The pion and the nucleon masses are both neglected, $p_{1}^{2}=0, p_{2}^{2}=\left(p_{2}^{\prime}\right)^{2}=0$. We denote the momenta of the outgoing quark and antiquark (jets) as $q_{1}$ and $q_{2}$, respectively. They are on the mass shell, $q_{1}^{2}=q_{2}^{2}=0$, and can be decomposed

$$
q_{1}=z p_{1}+\frac{q_{1 \perp}^{2}}{z s} p_{2}+q_{1 \perp}, q_{2}=\bar{z} p_{1}+\frac{q_{2 \perp}^{2}}{\bar{z} s} p_{2}+q_{2 \perp}
$$

such that $z$ is the longitudinal momentum fraction of the quark jet in the lab frame. We use the shorthand notation: $\bar{u} \equiv(1-u)$ for any longitudinal momentum fraction $u$.

We are interested in the forward limit, when the transferred momentum $t=\left(p_{2}-p_{2}^{\prime}\right)^{2}$ is equal to zero and the transverse momenta of jets compensate each other $q_{1 \perp} \equiv q_{\perp}, q_{2 \perp} \equiv-q_{\perp}$. In this kinematics the invariant mass of the produced $q \bar{q}$ pair is equal to $M^{2}=q_{\perp}^{2} / z \bar{z}$. The invariant c.m. energy $s=\left(p_{1}+p_{2}\right)^{2}=2 p_{1} p_{2}$ is taken to be much larger than the transverse jet momentum $q_{\perp}^{2}$. 
3. The possibility to constrain the pion distribution amplitude $\phi_{\pi}\left(z^{\prime}, \mu_{F}^{2}\right)$ in the dijet diffractive dissociation experiment assumes that the amplitude of this process can be calculated in the collinear approximation as suggested by Fig. 1:

$$
M_{\pi \rightarrow 2 \text { jets }}=\sum_{p=q, \bar{q}, g} \int_{0}^{1} d z^{\prime} \int_{0}^{1} d x_{1} \phi_{\pi}\left(z^{\prime}, \mu_{F}^{2}\right) T_{H}^{p}\left(z^{\prime}, x_{1}, \mu_{F}^{2}\right) F_{\zeta}^{p}\left(x_{1}, \mu_{F}^{2}\right) .
$$

Here $F_{\zeta}^{p}\left(x_{1}, \mu_{F}^{2}\right)$ is the generalized parton distribution (GPD) $p=q, \bar{q}, g$ $13,14,15$ in the target nucleon; $x_{1}$ and $x_{2}=x_{1}-\zeta$ are the momentum fractions of the emitted and the absorbed partons, respectively. The asymmetry parameter $\zeta$ is fixed by the process kinematics: $\zeta=M^{2} / s=q_{\perp}^{2} / z \bar{z}$. $T_{H}\left(z^{\prime}, x_{1}, \mu_{F}^{2}\right)$ is the hard scattering amplitude involving at least one hard gluon exchange and $\mu_{F}$ is the (collinear) factorization scale. By definition, the pion distribution amplitude only involves small momenta, $k_{\perp}<\mu_{F}$, and the hard scattering amplitude is calculated neglecting the parton transverse momenta.

We calculated ${ }^{10}$ both the leading-order gluon and quark contributions to the amplitude and find that in both cases the corresponding hard kernels $T_{H}^{q}, T_{H}^{g}$ diverge as $1 / z^{\prime 2}$ and $1 / \bar{z}^{\prime 2}$ in the $z^{\prime} \rightarrow 0$ and $z^{\prime} \rightarrow 1$ limit, respectively. This implies that the integration of the pion momentum fraction diverges at the end-points and the collinear factorization is, therefore, broken.

At high energies the contribution of gluon GPD dominates. We found that up to kinematical factors

$$
\begin{aligned}
T_{H}^{g}= & C_{F}\left(\frac{\bar{z}}{z^{\prime}}+\frac{z}{\bar{z}^{\prime}}\right)\left(\frac{\zeta}{\left[x_{1}-i \epsilon\right]^{2}}+\frac{\zeta}{\left[x_{2}+i \epsilon\right]^{2}}-\frac{\zeta}{\left[x_{1}-i \epsilon\right]\left[x_{2}+i \epsilon\right]}\right) \\
& +\left(\frac{z \bar{z}}{z^{\prime} \bar{z}^{\prime}}+1\right)\left[C_{F}\left(\frac{z \bar{z}}{z^{\prime} \bar{z}^{\prime}}+1\right)+\frac{1}{2 N_{c}}\left(\frac{z}{z^{\prime}}+\frac{\bar{z}}{\bar{z}^{\prime}}\right)\right] \\
& \times\left(\frac{1}{\left[\left(z-z^{\prime}\right) x_{1}-z \bar{z}^{\prime} \zeta+i \epsilon\right]}+\frac{1}{\left[\left(z^{\prime}-z\right) x_{2}-z \bar{z}^{\prime} \zeta+i \epsilon\right]}\right) \\
& -\left[C_{F} \frac{z \bar{z}}{z^{\prime} \bar{z}^{\prime}}\left(\frac{\bar{z}}{z^{\prime}}+\frac{z}{\bar{z}^{\prime}}\right)+\frac{1}{2 N_{c} z^{\prime} \bar{z}^{\prime}}\left(\frac{z \bar{z}}{z^{\prime} \bar{z}^{\prime}}+1\right)\right] \frac{\zeta}{\left[x_{1}+i \epsilon\right]\left[x_{2}-i \epsilon\right]}
\end{aligned}
$$

The differential cross section summed over the polarizations and the color of quark jets is given by

$$
\frac{d \sigma_{\pi \rightarrow 2 \mathrm{jets}}}{d q_{\perp}^{2} d t d z}=\frac{\alpha_{s}^{4} f_{\pi}^{2} \pi(1-\zeta)}{8 N_{c}^{3} q_{\perp}^{8}}|M|^{2}
$$

where $M$ is calculated as in (2) with $T_{H}^{g}$ given in (3), $f_{\pi}=133 \mathrm{MeV}$ is the pion decay constant. 
4. According to Eq. (3) the leading end-point behavior of the gluon amplitude at $z^{\prime} \rightarrow 0$ and $z^{\prime} \rightarrow 1$ is given by the following expression

$$
\left.M\right|_{\text {end-points }}=-i \pi\left(N_{c}+\frac{1}{N_{c}}\right) z \bar{z} \int_{0}^{1} d z^{\prime} \frac{\phi_{\pi}\left(z^{\prime}, \mu^{2}\right)}{z^{\prime 2}} F_{\zeta}^{g}\left(\zeta, \mu^{2}\right) .
$$

Since $\phi_{\pi}\left(z^{\prime}\right) \sim z^{\prime}$ at $z^{\prime} \rightarrow 0$, the integral over $z^{\prime}$ diverges logarithmically. Remarkably, the integral containing the pion distribution amplitude does not involve any $z$-dependence. Therefore, the longitudinal momentum distribution of the jets in the nonfactorizable contribution is calculable and, as it turns out, has the shape of the asymptotic pion distribution amplitude $\phi_{\pi}^{\mathrm{as}}(z)=6 z \bar{z}$.

In technical terms, the appearance of the end point divergency is due to pinching of the $x_{1}$ contour in the point $x_{1}=\zeta\left(x_{2}=0\right)$ in the case when variable $z^{\prime}$ is closed to the end points, cf. Eqs. $(2,3)$. One can trace ${ }^{10}$ that this pinching occurs between soft gluon (the gluon with momentum $x_{2} \rightarrow 0$ ) interactions in the initial and in the final state, and is related with the existence of the unitarity cuts of the amplitude in different, $s$ and $M^{2}$, channels.

The other important integration region in $(2)$ is the one when $\zeta \ll$ $\left|z^{\prime}-z\right| \ll 1$, i.e. when the longitudinal momentum fraction carried by the quark is close (for high energies) to that of the quark jet in the final state

$$
\begin{aligned}
\left.M\right|_{\zeta \ll\left|z^{\prime}-z\right| \ll 1} & =-4 i \pi N_{c} \phi_{\pi}(z) \int_{z}^{1} \frac{d z^{\prime}}{z^{\prime}-z} F_{\zeta}^{g}\left(\zeta \frac{z \bar{z}}{z^{\prime}-z}, q_{\perp}^{2}\right) \\
& \simeq-4 i \pi N_{c} \phi_{\pi}(z) \int_{\zeta}^{1} \frac{d y}{y} F_{\zeta}^{g}\left(y, q_{\perp}^{2}\right) .
\end{aligned}
$$

This logarithmic integral is nothing but the usual energy logarithm that accompanies each extra gluon in the gluon ladder. Its appearance is due to the fact the hard gluon which supplies jets by the high transverse momentum can be emitted in a broad rapidity interval and is not constrained to the pion fragmentation region. The integral on the r.h.s. of (6) can be identified with the unintegrated generalized gluon distribution. And, therefore, in the region $z^{\prime} \sim z$ hard gluon exchange can be viewed as a large transverse momentum part of the gluon distribution in the proton, cf. ${ }^{7}$. This contribution is proportional to the pion distribution amplitude $\phi_{\pi}\left(z, q_{\perp}^{2}\right)$ and contains the enhancement factor $\ln 1 / \zeta \sim \ln s / q_{\perp}^{2}$.

5. We performed numerical calculations for the kinematics of E791 experiment: the transverse momentum range $1.5 \leq q_{\perp} \leq 2.5 \mathrm{GeV}$ and 
$s=1000 \mathrm{GeV}^{2}$. We found that the diffractive (6) and the end-point (5) contributions are numerically comparable to each other. In the later case in order to regularize the end-point divergence we used the simplest prescription, an explicit cutoff on the quark momentum fraction in the pion $z^{\prime} \geq \mu_{\mathrm{IR}}^{2} / q_{\perp}^{2}$, where $\mu_{\mathrm{IR}}$ is of order of intrinsic quark transverse momentum in the pion, see ${ }^{10}$ for more details. For GPD's the parametrization ${ }^{16}$ was used. Fig. 2 shows the comparison of the calculated dijet momentum fraction distribution with the data ${ }^{4}$. The two solid curves correspond to the asymptotic, $\phi_{\pi}^{\mathrm{as}}(z)$, and 'two-humped", $\phi_{\pi}^{\mathrm{CZ}}(z, \mu=0.5 \mathrm{GeV})=30 z(1-z)(1-2 z)^{2}$, forms of the pion distribution amplitude. The dashed curve corresponds to the Chernyak-Zhitnitsky model evolved to the scale $\mu=2 \mathrm{GeV}$, $\phi_{\pi}^{\mathrm{CZ}}(z, \mu=2 \mathrm{GeV})=15 z(1-z)\left[0.20+(1-2 z)^{2}\right.$. The overall normalization is arbitrary, but is the same for all three choices of the distribution amplitude. It is seen that experimental uncertainties do not allow for the separation between the distribution amplitudes $\phi_{\pi}^{\text {as }}(z)$ and $\phi_{\pi}^{\mathrm{CZ}}(z, \mu=2 \mathrm{GeV})$ while the extreme choice $\phi_{\pi}^{\mathrm{CZ}}(z, \mu=0.5 \mathrm{GeV})$ is not favored. This general conclusion is in agreement with the analysis in ${ }^{12}$.

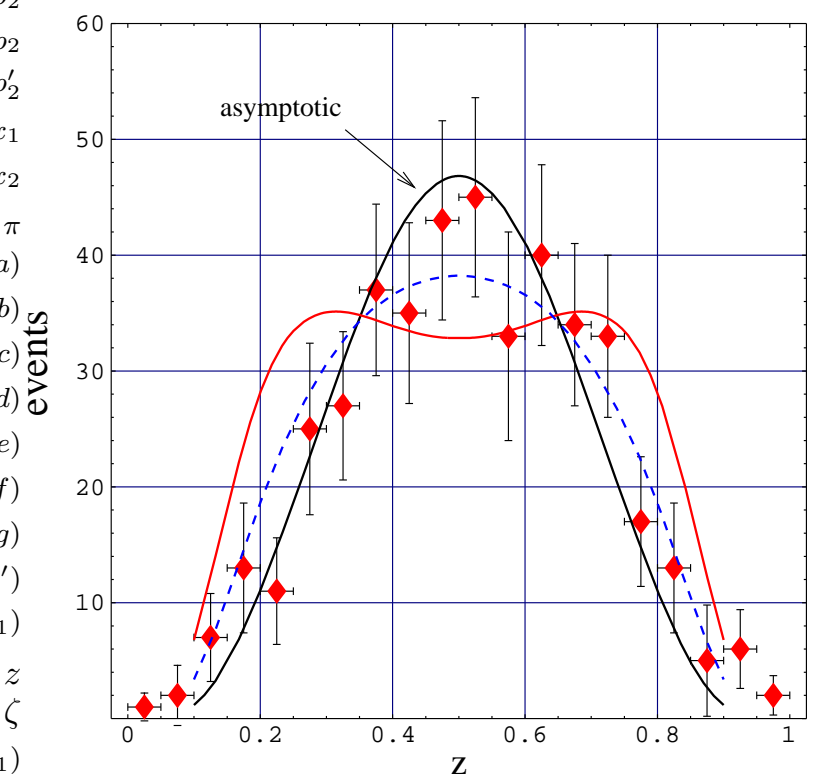

Figure 2. The longitudinal momentum fraction distribution of the dijets from the platinum target ${ }^{4}$ in comparison with our predictions, see text.

6. Our calculation is close in spirit to ${ }^{11,12}$ although the conclusion about 
the factorization is different. We examined the transition to the light-cone limit carefully and found that the approximation used in 11,12 breaks down for soft gluons (and quarks). In the double logarithmic approximation our result in (6) is similar to ${ }^{7}$ obtained using different methods. We, therefore, agree with the interpretation suggested in ${ }^{7}$ that in the true diffraction limit, for very large energies, the dijet production can be considered as a probe of the hard component of the pomeron. We note, however, that this interpretation breaks down beyond the double logarithmic approximation and is not sufficient for the energy region of the E791 experiment. Finally, we have to mention an approach to coherent diffraction suggested in ${ }^{8}$ that attributes hard dijets to a hard component of the pion wave function. This technique is interesting, but apparently complicated for the discussion of

factorization. We found that the general argumentation in ${ }^{8}$ appears to be in contradiction with our explicit calculations.

\section{Acknowledgments}

This work was supported by Alexander von Humboldt Foundation.

\section{References}

1. S. F. King, A. Donnachie and J. Randa, Nucl. Phys. B167 98 (1980); J. Randa, Phys. Rev. D22 1583 (1980).

2. G. Bertsch, S. J. Brodsky, A. S. Goldhaber and J. F. Gunion, Phys. Rev. Lett. 47297 (1981).

3. L. Frankfurt, G. A. Miller and M. Strikman, Phys. Lett. B 3041 (1993).

4. E. M. Aitala et al. [E791 Collaboration], Phys. Rev. Lett. 864768 (2001).

5. E. M. Aitala et al. [E791 Collaboration], Phys. Rev. Lett. 864773 (2001).

6. J. Gronberg et al. [CLEO Collaboration], Phys. Rev. D57 33 (1998).

7. N. N. Nikolaev, W. Schäfer and G. Schwiete, Phys. Rev. D63 014020 (2001).

8. L. Frankfurt, G. A. Miller and M. Strikman, Found. Phys. 30533 (2000) [hep-ph/9907214]; Phys. Rev. D65 094015 (2002) [hep-ph/0010297].

9. V. M. Braun, D. Yu. Ivanov, A. Schäfer and L. Szymanowski, Phys. Lett. B509 43 (2001).

10. V. M. Braun, D. Yu. Ivanov, A. Schafer and L. Szymanowski, arXiv:hep$\mathrm{ph} / 0204191$.

11. V. Chernyak, Phys. Lett. B516 (2001) 116.

12. V. L. Chernyak and A. G. Grozin, Phys. Lett. B517 119 (2001).

13. D. Müller, D. Robaschik, B. Geyer, F. M. Dittes and J. Horejsi, Fortsch. Phys. 42101 (1994); [arXiv:hep-ph/9812448].

14. A. V. Radyushkin, Phys. Lett. B385 333 (1996); Phys. Rev. D56 5524 (1997).

15. X. Ji, Phys. Rev. Lett. 78610 (1997); J. Phys. G. 241181 (1998).

16. A. Freund and M. F. McDermott, Phys. Rev. D65, 074008 (2002);

http://durpdg.dur.ac.uk/hepdata/dvcs.html 